PROCEEDINGS OF THE

AMERICAN MATHEMATICAL SOCIETY

Volume 129, Number 3, Pages $713-717$

S 0002-9939(00)05773-7

Article electronically published on November 8, 2000

\title{
ANALYTIC NORMS IN ORLICZ SPACES
}

\author{
P. HÁJEK AND S. TROYANSKI \\ (Communicated by Dale Alspach)
}

\begin{abstract}
It is shown that an Orlicz sequence space $h_{M}$ admits an equivalent analytic renorming if and only if it is either isomorphic to $l_{2 n}$ or isomorphically polyhedral. As a consequence, we show that there exists a separable Banach space admitting an equivalent $C^{\infty}$-Fréchet norm, but no equivalent analytic norm.
\end{abstract}

In this note, we denote by $h_{M}$ as usual the subspace of an Orlicz sequence space $l_{M}$ generated by the unit vector basis.

More terminology and notation concerning Orlicz spaces can be found in [LT].

Let us also point out that by $C^{k}$-smoothness (or analyticity) of a norm we always mean away from the origin (as is usual in renorming theory).

The characterization of the best order of $C^{k}$-Fréchet smoothness of some renorming, $k \in \mathbb{N} \cup\{+\infty\}$, for $h_{M}$ was obtained in [M], [MT1, [MT2]. In our present note, we complete the characterization also for analytic renormings. We show that an Orlicz sequence space $h_{M}$ has an analytic renorming if and only if $h_{M} \cong l_{2 n}$, $n \in \mathbb{N}$, or $h_{M}$ is isomorphically polyhedral. Let us recall that a separable Banach space $X$ is isomorphically polyhedral if it has an equivalent polyhedral norm. By a theorem of Fonf $[\mathrm{F}$, this is the case if and only if $X$ admits an equivalent norm with a countable boundary. More precisely, there exists a sequence $\left\{f_{i}\right\}_{n \in \mathbb{N}}$ in $X^{*}$ such that

$$
\|x\|=\max \left\{\left|f_{i}(x)\right|, \quad i \in \mathbb{N}\right\} .
$$

According to one of the results from $[\mathrm{DFH}]$, we have the following:

Theorem 1. Every separable isomorphically polyhedral Banach space $X$ admits an equivalent analytic form.

We prove that the converse is also true if we impose additional conditions on the space $X$. In connection with our result it should be noted that by recent work of Gonzalo and Jaramillo (GJ) every separable Banach space with a symmetric basis and $C^{\infty}$-Fréchet smooth norm is isomorphic to $l_{2 n}$, provided it does not contain a copy of $c_{0}$.

Our approach is entirely different from that in [MT1] and relies on methods from [DFH] and [H1]- $\mathrm{H3}$ ]. As a corollary, relying on an example of Leung [L], we show that there exists a separable Banach space with $C^{\infty}$-Fréchet smooth norm which

Received by the editors March 30, 1998 and, in revised form, November 25, 1998.

2000 Mathematics Subject Classification. Primary 46B03, 46B45.

Key words and phrases. Analytic norm, Orlicz space, polyhedral space.

The second author was partially supported by NFSR of Bulgaria, Grant MM-808-98.

(C)2000 American Mathematical Society 
admits no analytic norm. A search of such an example was in fact a motivation of our work, since the previously known examples of such spaces (e.g. $c_{0}(\Gamma), \Gamma$ uncountable; see $[\mathrm{P}]$ and $[\mathrm{BF}]$ for a result of Kuiper) were nonseparable.

Let us recall that a Banach space $X$ with an unconditional basis is said to satisfy an upper $p$-estimate, $p \geq 1$, if for some $C>0$ :

$$
\left\|\sum_{i=1}^{n} u_{i}\right\| \leq C\left(\sum_{i=1}^{n}\left\|u_{i}\right\|^{p}\right)^{\frac{1}{p}}
$$

whenever $u_{i}$ are disjointly supported in $X$.

An important notion in our consideration is that of weak sequential continuity.

Definition 2. Let $\mathcal{U} \subseteq X$ be an open, convex and bounded subset of a Banach space $X, f$ be a real function on $\mathcal{U}$. We say that $f$ is weakly sequentially continuous (wsc-for short) if it maps weakly Cauchy sequences from $\mathcal{U}$ into convergent ones. A function $f$ defined on an open subset $\mathcal{O} \subseteq X$ is said to be locally wsc if there exists a covering of $\mathcal{O}$ by a family of open sets $\mathcal{U}$ as above such that $f$ is wsc on $\mathcal{U}$ for all $\mathcal{U}$.

In order to verify wsc-property for polynomials, it is sufficient to check the convergence only for weakly convergent sequences in $\mathcal{U}$ ([AHV]).

Using this fact, the following lemma follows from results in $G$.

Lemma 3. Let $X$ be a Banach space with an unconditional basis satisfying an upper $p$-estimate. Then all polynomials of degree $n<p$ on $X$ are wsc (on $B_{X}$ ).

The importance of the notion of wsc stems from the following lemma, which comes from [H3], and which was shown for polynomials in [AHV].

Lemma 4. Let $X$ be a Banach space $l_{1} \nrightarrow X, f$ be a $C^{2}$-Fréchet differentiable real function defined on some open set $\mathcal{O} \subseteq X$. TFAE:

(1) $f$ is locally wsc,

(2) $f^{\prime}$ is locally norm compact.

By $f^{\prime}$ being locally norm compact we mean that there exists a covering by a family of open sets $\mathcal{U}$ of $\mathcal{O}$ such that $f^{\prime}(\mathcal{U})$ is relatively norm compact in $X^{*}$ for all $\mathcal{U}$.

The following is a generalization of the main result in [H1].

Theorem 5. Let $(X,\|\cdot\|)$ be a Banach space, where $\|\cdot\|$ is analytic. If all polynomials on $X$ are wsc, then $X$ is separable and isomorphically polyhedral.

Proof. By $\partial$ we denote the duality map corresponding to $\|\cdot\|$, i.e.

$$
\partial: X \backslash\{0\} \rightarrow S_{X^{*}} \quad \text { and } \quad \partial x(x)=\|x\| \quad \text { for all } x \in X \backslash\{0\} .
$$

Since $\|\cdot\|$ is differentiable, $\partial x$ is the derivative of $\|x\|$ at $x \in X \backslash\{0\}$.

Let us first show that $\|\cdot\|$ is locally wsc on $X \backslash\{0\}$.

Fix $x \neq 0$. Since $\|\cdot\|$ is analytic at $x$ we can find $\delta>0$ so that if $\|h\|<\delta$ we have

$$
\|x+h\|=\sum_{n=1}^{\infty} p_{n}(h)
$$


where $p_{n}$ are homogeneous polynomials of degree $n$, and the convergence is uniform with respect to all $\|h\|<\delta$. Since all polynomials $p_{n}$ are wsc, we see that $\|\cdot\|$ is wsc on $\{y:\|x-y\|<\delta\}$.

We proceed by showing that $X$ is separable. Since $X$ is an Asplund space, from Lemma 4 we get that there exist $0<\eta<\delta$ so that the set $\{\partial y:\|x-y\|<\eta\}$ is norm relatively compact. Thus the subspace $Y$ of $X^{*}$ generated by $\{\partial y:\|y-x\|<\eta\}$ is separable. If $Y=X^{*}$ the proof is finished. Otherwise, assume $Y \neq X^{*}$. By the Hahn-Banach theorem, there exists $x^{* *} \in S_{X^{* *}}$ such that

$$
x^{* *}(\partial y)=0 \quad \text { whenever } \quad\|y-x\|<\eta .
$$

Since $\|\cdot\|$ is analytic on $X \backslash\{0\}$ we get that $\partial$ is analytic as well on $X \backslash\{0\}$. Hence $f=x^{* *} \circ \partial$ is a real analytic function on $X \backslash\{0\}$.

Since $f(y)=0$ for $\|y-x\|<\eta$, clearly $f \equiv 0$ on $X \backslash\{0\}$. On the other hand, by the Bishop-Phelps theorem, $\partial S_{X}$ is dense in $S_{X^{*}}$, so there exists $y \in S_{X}$ such that $f(y)=x^{* *}(\partial y) \neq 0$, a contradiction. So $X$ is separable.

Since $X$ is separable and $\|\cdot\|$ is locally wsc, by Lemma 4 and the Lindelöf property, $\left(S_{X},\|\cdot\|\right)$ can be covered by a countable system $\left\{\mathcal{U}_{n}\right\}_{n \in \mathbb{N}}$ of norm open convex bounded subsets of $X$ such that $\partial \mathcal{U}_{n}$ is relatively compact. Thus the boundary of $(X,\|\cdot\|)$ can be covered by a countable system of compacts, and the result follows from [H2].

Theorem 6. Let $M$ be an Orlicz function. Then $h_{M}$ admits an equivalent analytic norm if and only if either $h_{M} \cong l_{2 n}, n \in \mathbb{N}$, or $h_{M}$ is isomorphically polyhedral. In particular if

(1) $\lim _{t \rightarrow 0} \frac{M(2 t)}{M(t)}=+\infty$, then $h_{M}$ has an equivalent analytic norm.

(2) $a_{M}=+\infty$ and there exists a sequence $t_{i} \searrow 0$ such that $\sup _{i \in \mathbb{N}} \frac{M\left(a t_{i}\right)}{M\left(t_{i}\right)}<+\infty$ for all $a \geq 1$, then $h_{M}$ does not admit an equivalent analytic norm.

Proof. The "if" part follows from the well-known result that the canonical norm on $l_{2 n}, n \in \mathbb{N}$, is analytic and from Theorem 1 .

The "only if" part: By classical results ([LT] $)$, the existence of an analytic norm on $X$ implies $\alpha_{M}=\beta_{M} \in\{2 n\}_{n \in \mathbb{N}} \cup\{+\infty\}$.

The case $\alpha_{M}=2 n$ implies that $X \cong l_{2 n}$ by [MT1].

If $\alpha_{M}=\infty$, then ([LT] $X$ has an upper $p$-estimate for every $p>1$.

Combination of Lemma 3 and Theorem 5 finishes the proof of the "only if" part.

Leung [ $\mathrm{L}$ ] showed that if $M$ satisfies (1), then $h_{M}$ is isomorphically polyhedral and if $M$ satisfies (2) $h_{M}$ is not isomorphically polyhedral.

Corollary 7. There exists a $c_{0}$-saturated separable Banach space which admits an equivalent $C^{\infty}$-Fréchet norm but no equivalent analytic norm.

Proof. Leung [L] constructed an Orlicz function $M$ satisfying (2). By a result of [MT2 the corresponding space $h_{M}$ admits an equivalent $C^{\infty}$-Fréchet smooth norm. On the other hand, by Theorem [6] no equivalent analytic norm exists on this space.

Let us pass to some final remarks. A natural question is the following: Is there a separable $c_{0}$-saturated non-polyhedral Banach space with an equivalent analytic norm? 
By a careful analysis of $[\overline{D F H}$, we obtain that on every separable polyhedral space there exists a dense set of equivalent analytic norms whose boundaries can be covered by countably many compacts. Such norms in turn immediately imply the polyhedrality of the space (using [H2]).

However, there are examples of polyhedral spaces (e.g. $[\underline{\mathrm{S}}],[\mathrm{PS}]$ ) with analytic norms failing this property.

More precisely, the space $S$ of Schreier has an unconditional basis $\left\{e_{n}\right\}$ such that the formal identity operator id from $S$ into $l_{2}$ is bounded. It is easy to show that given an equivalent analytic norm $\|\cdot\|$ on $S$ whose boundary is covered by countably many compacts, the equivalent analytic norm

$$
\|x\|=\left(\|x\|^{2}+\|\operatorname{id} x\|_{2}^{2}\right)^{\frac{1}{2}}
$$

fails the covering property.

The problem is therefore how to recognize the polyhedrality of $S$ based on its norm $\|\cdot\| \cdot$

\section{ACKNOWLEDGEMENT}

The authors would like to thank the Department of Analysis of Universidad Complutense in Madrid, for hospitality and excellent working conditions during the preparation of this note.

\section{REFERENCES}

[AHV] R. Aron, C. Hervés and M. Valdivia, Weakly continuous mappings on Banach spaces, J. Funct. Anal. 52 (1983), 189-204. MR 84g:46066

[BF] R. Bonic and J. Frampton, Smooth functions on Banach manifolds, J. of Math. and Mech. 15 (1966), 877-898. MR 33:6647

[D] R. Deville, Geometrical implications of the existence of very smooth bump functions in Banach spaces, Israel J. Math. 67 (1989), 1-22. MR 90m:46023

[DFH] R. Deville, V. Fonf and P. Hájek, Analytic and polyhedral approximation of convex bodies in separable polyhedral Banach spaces Israel J. Math. 105 (1998), 139-154. MR 99h:46006

[F] V. Fonf, One property of Lindenstrauss-Phelps spaces, Functional Anal. Appl. 13 (1979), 66-67.

[G] R. Gonzalo, Upper and lower estimates in Banach sequence spaces, Comment Math. Univ. Carolinae 36 (1995), 641-653. MR 97d:46020

[GJ] R. Gonzalo and J. A. Jaramillo, Smoothness and estimates of sequences in Banach spaces, Israel J. Math. 89 (1995), 321-341. MR 96h:46017

[H1] P. Hájek, Analytic renormings of $C(K)$ spaces, Serdica Math. J. 22 (1996), 25-28. MR 97h:46014

[H2] P. Hájek, Smooth norms that depend locally on finitely many coordinates, Proc. Amer. Math. Soc. 123 (1995), 3817-3821. MR 96e:46017]

[H3] P. Hájek, Smooth functions on $C(K)$, Israel J. Math. 107 (1998), 237-252. MR 99k:46072

[L] D. H. Leung, Some isomorphically polyhedral Orlicz sequence spaces, Israel J. Math. 87 (1994), 117-128. MR 95f:46033

[LT] J. Lindenstrauss and R. R. Tzafriri, Classical Banach Spaces I: Sequences Spaces, SpringerVerlag, New York, 1977. MR 54:3344

[M] R. P. Maleev, Norms of best smoothness of Orlicz spaces, Z. Anal. Anwendungen 12 (1993), 123-135. MR 94m:46049

[MT1] R. P. Maleev and S. L. Troyanski, Smooth functions in Orlicz spaces, Contemp. Math. 85 (1989), 336-351. MR 90a:46034

[MT2] R. P. Maleev and S. L. Troyanski, Smooth norms in Orlicz spaces, Canad. Math. Bul. 34 (1) (1991), 74-82. MR 92j:46052

[P] A. Pelczynski, A property of multilinear operations, Studia Math. 16 (1957), 173-182. MR 20:221 
[PS] A. Pelczynski and W. Szlenk, An example of a non-shrinking basis, Rev. Roumaine Math. Pures Appl. 10 (7) (1965), 961-966. MR 34:3284

[S] J. Schreier, Ein Gegenbeispiel zur Theorie der schwachen Konvergenz, Studia Math. 2 (1930), 58-62.

Departamento Análisis Matemático, Universidad Complutense de Madrid, 28040 Madrid, Spain and Mathematical Institute, Czech Academy of Science, Žitná 25, Prague, Czech Republic

Current address: Department of Mathematics, Texas A\&M University, College Station, Texas 77843

E-mail address: phajek@math.tamu.edu

Departmento Análisis Matemático, Universidad Complutense de Madrid, 28040 Madrid, Spain and Department of Mathematics and Informatics, Sofia University, 5, James Bourchier Blvd., 1126 Sofia, Bulgaria 